\title{
Intelligent Decision Making Using Soft Computing
}

\author{
Ronald R. Yager \\ Machine Intelligence Institute \\ Iona College \\ New Rochelle, NY 10801 \\ yager@panix.com
}

\begin{abstract}
Intelligent decision making requires one to contend with a number of difficult aspects. The need to satisfy multiple criteria in selecting a solution is pervasive in many real world decision problems. An additional feature that must be addressed is the uncertainty and riskiness due to our lack of complete knowledge of the future and the effects of our actions. Further compounding the decision making environment is the need to take into account the effects of the actions of other agents who may being working in an adversarial way. Another important requirement in intelligent decision making is the ability to model the preferences of the responsible decision maker. During this talk we shall describe some of the tools that fuzzy sets and other soft computing technologies can bring to bear on this problem. Here we shall particularly focus on the issue of multi-criteria.
\end{abstract}

